\title{
Ultrasound Mediated Delivery of Liposomal Doxorubicin in Mice with Glioma
}

\author{
Feng-Yi Yang ${ }^{1}$, Shih-Cheng Horng ${ }^{2}$ \\ ${ }^{1}$ Department of Biomedical, Imaging and Radiological Sciences, School of Biomedical Science and Engineering, \\ National Yang-Ming University, Taipei, Taiwan \\ ${ }^{2}$ Department of Computer Science \& Information, Engineering, Chaoyang University of Technology, Taichung, Taiwan \\ Email: fyyang@ym.edu.tw, schong@cyut.edu.tw
}

Received 2012

\begin{abstract}
Malignant brain tumors remain difficult to treat with chemotherapy because the blood-brain barrier (BBB) limits the amounts of potent agents that can reach the tumor, such that the drugs are unable to reach therapeutic dosage. Although various targeted carriers that encapsulate chemotherapeutic agents have been shown to improve drug delivery to brain tumors, the BBB is still a major obstacle in the use of chemotherapy for the treatment of these tumors. Human glioblastoma-bearing mice were injected intravenously with doxorubicin (Dox) encapsulated in atherosclerotic plaque-specific peptide-1 (AP-1)-conjugated liposomes or unconjugated liposome. These treatments took place with or without BBB disruption induced by transcranial pulsed high-intensity focused ultrasound (pulsed HIFU). This study showed that the treatment with Dox encapsulated in AP-1-conjugated liposomes followed by pulsed HIFU enhanced the accumulation of the cytotoxic drug in cells and inhibited the growth of brain tumors in vivo. Combining pulsed HIFU with cytotoxic agents might improve their efficacy in patients with brain tumors while simultaneously reducing the drug side effects. Further investigation is required to provide a comprehensive physical characterization of the sonication process and to determine its bioeffects.
\end{abstract}

Keywords: Focused Ultrasound; IL-4; Blood-brain Barrier; Brain Tumor; Drug Delivery

\section{Introduction}

The limited amount of chemotherapeutic agent present in the circulation is able to be transported into brain tumors without the assistance of a blood-tumor barrier (BTB) delivery system due to the presence of the BTB. Some works have reported that using infusion of hyperosmotic solution of mannitol, which disrupts the BTB, the drug uptake in brain tumor could be higher than in tumor without BTB disruption [1-3]. Liposome-based drug delivery systems have been designed to increase tumor drug levels while limiting systemic drug dosage [4]. It is thought that the employment of liposomes conjugated to antibodies or targeting ligands can enhance the accumulation and retention of drugs at the tumor region compared with the free drug [5].

The glioblastoma multiforme (GBM) is one of the most common forms of glioma. It is hard to treat gliomas completely by surgical resection and therefore radiotherapy and chemotherapy are used to remove residual microscopic tumor material [6]. Previous reports have demonstrated that high-dose chemotherapy may have a potential survival benefit compared to historical controls treated with standard-dose therapy [7-8]. It has been shown that human brain tumor cell lines express high levels of plasma membrane interleukin-4 receptors (IL-4R) [9]. Thus, selective drug delivery may be achievable by binding chemotherapeutic agents to IL-4R [10]. A ligand from atherosclerotic plaque-specific peptide-1 (AP-1) was selected from phage display libraries that can locate atherosclerotic plaque tissue and bind to the IL-4 receptor, since it has the same bind- ing motif to the IL-4 protein. AP-1-labeled nanoparticles have been used for the targeted drug delivery to tumor in the previous works [11-12].

The purpose of this study was to demonstrate that the technology combines pulsed HIFU and liposomal nanoparticles as a synergistic delivery system for treating malignant brain tumors.

\section{Methodology}

\subsection{Glioma Xenograft Model}

Male 6 to 8-week-old NOD-scid mice were anesthetized by an intraperitoneal administration of pentobarbital at a dose of 40 $\mathrm{mg} / \mathrm{kg}$ of body weight. All animal experiments were performed according to the appropriate guidelines and approved by our Animal Care and Use Committee. The $2 \times 10^{5}$ Human brain malignant glioma (GBM8401) cells in $2 \mu \mathrm{L}$ culture medium were injected into the brains of the mice. Magnetic Resonance imaging (MRI) was used to determine that a tumor was established.

\subsection{Ultrasound Equipment}

Pulsed HIFU exposures were generated by a 1.0-MHz, single-element focused transducer (A392S, Panametrics, Waltham, MA, USA) with a diameter of $38 \mathrm{~mm}$ and a radius of curvature of $63.5 \mathrm{~mm}$. The focal zone of the therapeutic transducer was in the shape of an elongated ellipsoid, with a radial diameter (-6 $\mathrm{dB})$ of $3 \mathrm{~mm}$ and an axial length $(-6 \mathrm{~dB})$ of $26 \mathrm{~mm}$. The transducer with removable cone was fixed on a stereotaxic apparatus 
(Stoelting, Wood Dale, IL, USA) that permitting submilimeter placement of the ultrasound focus at the target location in the brain. A function generator (33220A, Agilent Inc., Palo Alto, USA) was connected to a power amplifier (500-009, Advanced Surgical Systems, Tucson, AZ) to drive the FUS transducer and a power meter/sensor module (Bird 4421, Ohio, USA) was used to measure the input electrical power. The rat's head was mounted on the stereotaxic apparatus with the nose bar positioned $3.3 \mathrm{~mm}$ below the interaural line. UCA (SonoVue, Bracco International, Amsterdam, The Netherlands) was injected into the femoral vein of the rats about $15 \mathrm{~s}$ before each sonication. The UCA contains phospholipid-coated microbubbles with a mean diameter $=2.5 \mu \mathrm{m}$, and at a concentration of 1 x $10^{8}$ to $5 \times 10^{8}$ bubbles $/ \mathrm{ml}$. Sonication was pulsed with a burst length of $50 \mathrm{~ms}$ at a $5 \%$ duty cycle and a repetition frequency of $1 \mathrm{~Hz}$. The duration of the sonication was $60 \mathrm{~s}$. The ultrasound beam was delivered to one location in the left brain hemisphere, centered on the tumor injection site. The following sonication parameters were used: an acoustic power of $2.86 \mathrm{~W}$ with an injection of $300 \mu \mathrm{l} / \mathrm{kg}$ UCA, a pulse repetition frequency of $1 \mathrm{~Hz}$, and a duty cycle of $5 \%$.

\subsection{Preparation of Liposomal Doxorubicin}

Lipo-Dox was prepared using a solvent injection method plus remote loading procedures. Briefly, hydrogenated soybean l- $\alpha-$ phosphatidylcholine (95.8 mg, Avanti Polar Lipids), cholesterol (31.9 mg, Sigma-Aldrich), and 1,2-distearoyl-sn-glycero-3phosphoethanolamine-N-[methoxy(polyethylene glycol)-2000] (DSPE-PEG2000, 31.9 mg, Avanti Polar Lipids) were dissolved and well mixed in $1 \mathrm{ml}$ of absolute ethanol at $60^{\circ} \mathrm{C}$. The lipid and ethanol mixture was then injected into a 9-ml solution of $250 \mathrm{mM}$ ammonium sulfate and stirred for $1 \mathrm{~h}$ at $60^{\circ} \mathrm{C}$. The mixture was then extruded five times through polycarbonate membranes (Isopore Membrane Filter, Millipore) with pore sizes of $0.4,0.2,0.1$, and $0.05 \mu \mathrm{m}$, consecutively, at $60^{\circ} \mathrm{C}$ with high-pressure extrusion equipment (Lipex Biomembranes) to produce small liposomes. The liposome suspension was then dialyzed five times against large amounts of $10 \%$ sucrose containing $5 \mathrm{mM} \mathrm{NaCl}$ to remove the unentrapped ammonium sulfate and ethanol. After dialysis, the liposome suspension was placed in a $50-\mathrm{ml}$ glass bottle in a $60^{\circ} \mathrm{C}$ water bath and mixed with Dox, to a final Dox concentration of $2 \mathrm{mg} / \mathrm{ml}$ in $10 \%$ sucrose solution. The bottle was intermittently shaken in a $60^{\circ} \mathrm{C}$ water bath for $1 \mathrm{~h}$ and then immediately cooled down to $4^{\circ} \mathrm{C}$, culminating in the production of Lipo-Dox.

Due to the presence of a thiol group on each cystine of the AP-1 peptide (CRKRLDRNC), it is possible to couple AP-1 to liposomes via the thiol-maleimide reaction. Briefly, AP-1 peptide was conjugated to 1,2-distearoyl-sn-glycero-3-phosphoethanolamine-N-[maleimid e(polyethylene glycol)-2000] (DSPE-PEG2000-MAL, Avanti Polar Lipids) by adding AP-1 to the DSPE-PEG2000-MAL micelle solution at a 2:1 molar ratio while mixing at $4^{\circ} \mathrm{C}$ overnight. The free thiol groups were measured with 5,5'-dithiobis-(2-nitrobenzoic acid) (Ellman's reagent, Sigma-Aldrich) at $420 \mathrm{~nm}$ to confirm that most of the AP-1 was conjugated with DSPE-PEG2000-MAL after the reaction. AP-1-conjugated DSPE-PEG2000 was transferred into the pre- formed Lipo-Dox at a $1.5 \%$ molar ratio of total lipid components and incubated at $60^{\circ} \mathrm{C}$ for $1 \mathrm{~h}$ to obtain AP-1-labeled Lipo-Dox (AP-1 Lipo-Dox; Figure 1).

\subsection{Quantitative Evaluation of Doxorubicin}

The procedures of single and repeated sonication are shown in Figure 2. The concentration of liposomes administered to the mice via tail-vein injection corresponded to $5 \mathrm{mg} / \mathrm{kg}$. The brain was perfused by transcardiac methods with normal saline $3.5 \mathrm{~h}$ after the Dox administration in order to flush unabsorbed Dox from the cerebral vessels. The site of tumor tissue was harvested along with its contralateral counterpart as a control. The concentration of Dox present was measured using a spectrophotometer (PowerWave 340, BioTek, USA; excitation at 480 $\mathrm{nm}$ and emissions measured at $590 \mathrm{~nm}$ ), with the value determined by taking the average of at least three fluorometric readings. The Dox present in the tissue samples was quantified using a linear regression standard curve derived from seven different concentrations of Dox; the amount of Dox was quantified as the absorbance per gram of tissue.

\subsection{Treatment Protocol}

A group of control mice was injected with GBM8401 glioma cells, but received no treatment. Five and 9 days after tumor cell implantation, one group of glioma-bearing mice received one of the following: (1) Lipo-Dox, or (3) AP-1 Lipo-Dox followed by single sonication. Another group of glioma-bearing mice treated Lipo-Dox or AP-1 Lipo-Dox followed by repeated sonication on day 5 after tumor implantation.

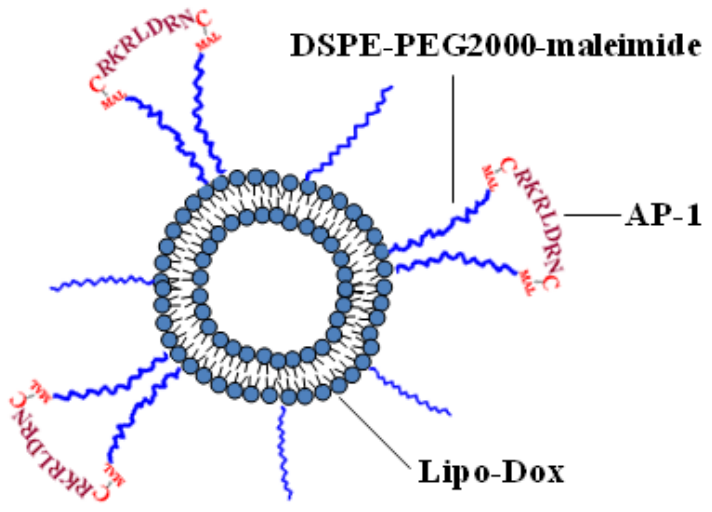

Figure 1. Schematic diagram for the AP-1 liposomal doxorubicin. Liposomes were prepared containing maleimide-functionaled polyethylene glycol chains. The maleimide was used to attach the AP-1 peptide through the thiol group on a cystine.

A

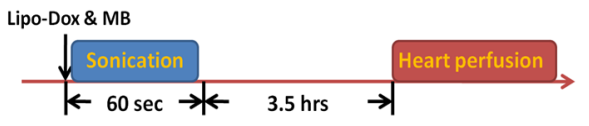

B

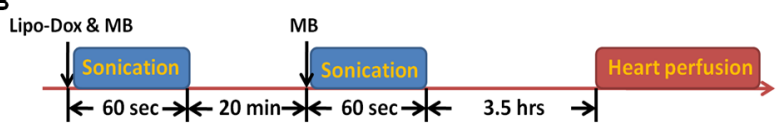


Figure 2. Diagrams of the experimental time line for (A) single sonication and (B) repeated sonication.

\subsection{MR Imaging}

Magnetic resonance imaging (MRI) was performed using a 3-T MRI system (TRIO 3-T MRI, Siemens MAGNETOM, Germany) after focused ultrasound sonication. The mice were anesthetized with isoflurane mixed with oxygen during the imaging procedure. A loop coil (Loop Flex Coil, approximately $4 \mathrm{~cm}$ in diameter) was used for RF reception. The imaging plane was located across the center of the tumor injection site. Tumor progression was monitored by means of T2-weighted images obtained from 4 to 16 days after tumor implantation. The parameters for T2-weighted imaging were as follows: repetition time/echo time $=3500 / 75 \mathrm{~ms}$, matrix $=125 \times 256$, field of view $=25 \times 43 \mathrm{~mm}$, and section thickness $=1.0 \mathrm{~mm}$

\section{Results}

Figure 3 shows the mean concentration of Dox per unit mass for the contralateral normal brain, the brain tumors and the brain tumors with single or repeated sonication after unconjugated Lipo-Dox or AP-1 Lipo-Dox administration.

The concentration of Dox was not only significantly greater in the unsonicated tumor BBB than in the contralateral normal brain region, but it was also significantly greater at the tumor site after single or repeated sonication than in the unsonicated tumor for the two forms of Lipo-Dox, especially for the repeated sonication. In addition, the concentration of Dox was significantly greater at the tumor site with unconjugated Lipo-Dox followed by single or repeated sonication than in the unsonicated tumor treated with AP-1 Lipo-Dox without sonication.

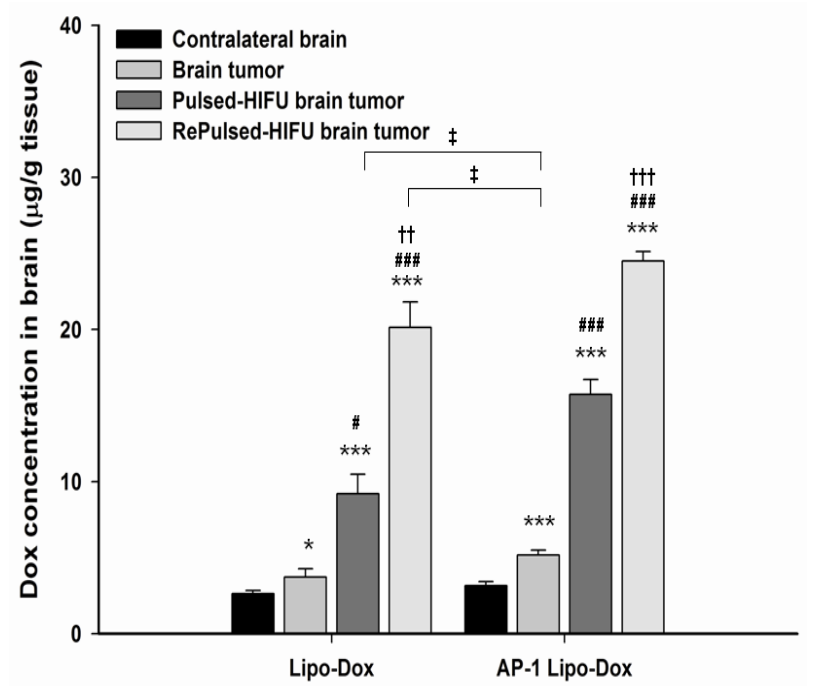

Figure 3. Measurements of Lipo-Dox and AP-1 Lipo-Dox in contralateral normal brain, the brain tumor and the brain tumor with single or repeated sonication. Compared with the contralateral normal brain, there was a significant difference for the control and sonicated tumors between the two forms of the drug. The concentrations of Dox were significantly higher in sonicated brain tumors than in unsonicated brain tumors. */***,\#/\#\#\# and ††/††† denote significant differences compared with the contralateral normal brain, the unsonicated brain tumor and single sonicated tumor,

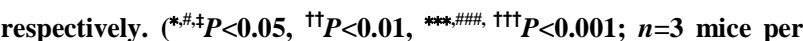
group).

Compared to the control tumor, there was a significant increase in the derived tumor-to-contralateral brain ratios for the single or repeated sonicated tumor treated with either drug (Figure 4). Interestingly, the derived tumor-to-contralateral brain ratio was significantly greater after single or repeated sonication for the untargeted Lipo-Dox group than for the targeted Lipo-Dox group without sonication.

Figure 5 shows the effects of the various treatment protocols on tumor growth were monitored by MR imaging on day 12 after implantation. These results suggest that AP-1 Lipo-Dox enhanced by pulsed HIFU at the tumor site is more effective at inhibiting tumor growth than AP-1 Lipo-Dox alone.

\section{Discussion}

Malignant glioma remains one of the most deadly types of tumor in humans, and GBM is one of the most common forms of glioma. Pulsed HIFU induced high-dose chemotherapy may have a potential survival benefit compared to historical controls treated with standard-dose therapy. This study showed that the treatment with Dox encapsulated in AP-1-conjugated liposomes followed by single or repeated pulsed HIFU enhanced the accumulation of the cytotoxic drug in cells and inhibited the growth of brain tumors in rats. Combining pulsed HIFU with cytotoxic agents might improve their efficacy in brain tumors with minimal systemic toxicity. This technology, employed with recent advances in targeted microbubbles, may promote

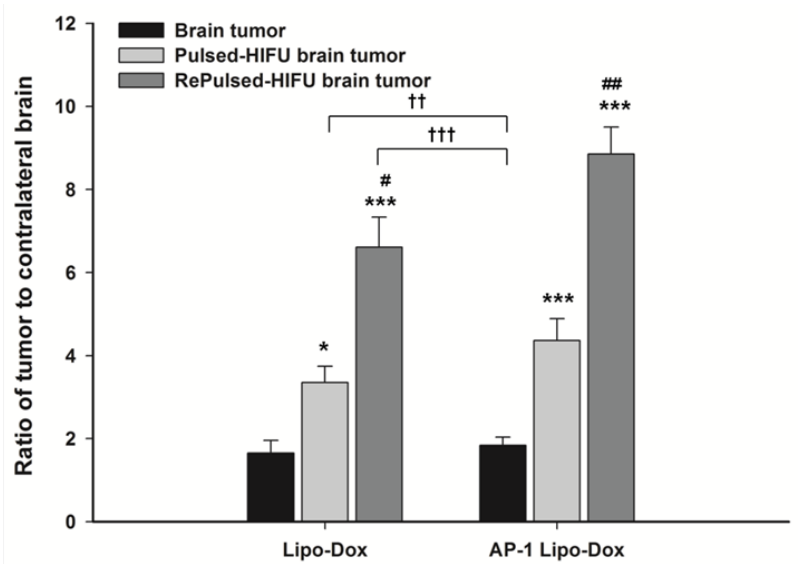

Figure 4. The derived tumor-to-contralateral brain ratios with unsonication, single sonication and repeated sonication after drug administration. $\left({ }^{*, H} P<0.05,{ }^{\#,+\dagger} P<0.01,{ }^{* * *,},{ }^{+\dagger \dagger} P<0.001 ; n=3\right.$ mice per group).

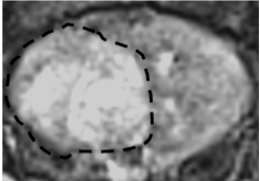

Control

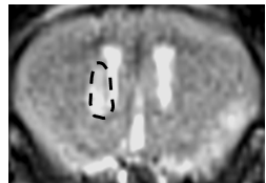

AP-1 Lipo-Dox

/Pulsed-HIFU
AP-1 Lipo-Dox

/RePulsed-HIFU

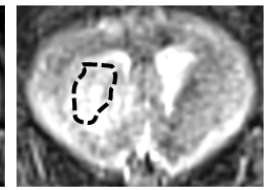

Figure 5. Representative sample of T2-weighted magnetic resonance imaging of a human GMB 8401 xenograft on day 12 after implantation. Compared to the control tumor, there was a clear 
decrease in the size of the tumor in mice treated with AP-1 LipoDox followed by single pulsed HIFU or repeated pulsed HIFU. new approaches to be developed that make targeted brain tumor therapy possible. The results of this pilot study therefore suggest it would provide targeted access for chemotherapy and allow the use of recombinant pharmaceuticals for the brain diseases.

\section{Acknowledgements}

This study was supported by grants from the National Science Council of Taiwan (no. NSC 100-2321-B-010-010 and NSC 99-2321-B-010-017), Cheng Hsin General Hospital Foundation (no. 100F117CY25), Veterans General Hospitals University System of Taiwan Joint Research Program (\#VGHUST100-G13-3 and V100E6-007), Yen Tjing Ling Medical Foundation (grant CI-100-17), Department of Health of Taiwan (DOH101TD-PB-111-TM012).

\section{REFERENCES}

[1] R. Barth, et al., "Boron neutron capture therapy of brain tumors: enhanced survival following intracarotid injection of either sodium borocaptate or boronophenylalanine with or without blood-brain barrier disruption," Cancer Research, vol. 57, p. 1129, 1997.

[2] R. F. Barth, et al., "Neutron capture therapy of intracerebral melanoma: enhanced survival and cure after blood-brain barrier opening to improve delivery of boronophenylalanine," Int J Radiat Oncol Biol Phys, vol. 52, pp. 858-68, Mar 12002.

[3] C. H. Hsieh, et al., "Evaluation of pharmacokinetics of 4-borono-2-(18)F-fluoro-L-phenylalanine for boron neutron capture therapy in a glioma-bearing rat model with hyperosmolar blood-brain barrier disruption," J Nucl Med, vol. 46, pp. 1858-65, Nov 2005.

[4] T. M. Allen and P. R. Cullis, "Drug delivery systems: entering the mainstream," Science, vol. 303, pp. 1818-22, Mar 192004.

[5] C. R. Dass, et al., "Enhanced anticancer therapy mediated by specialized liposomes," J Pharm Pharmacol, vol. 49, pp. 972-5, Oct 1997.

[6] D. F. Deen, et al., "Brain Tumor Working Group Report on the 9th International Conference on Brain Tumor Research and Therapy. Organ System Program, National Cancer Institute," J Neurooncol, vol. 16, pp. 243-72, Jun 1993.

[7] R. J. Motzer, et al., "Phase II trial of high-dose carboplatin and etoposide with autologous bone marrow transplantation in first-line therapy for patients with poor-risk germ cell tumors," J Natl Cancer Inst, vol. 85, pp. 1828-35, Nov 171993.

[8] R. J. Motzer, et al., "High-dose carboplatin, etoposide, and cyclophosphamide with autologous bone marrow transplantation in first-line therapy for patients with poor-risk germ cell tumors," J Clin Oncol, vol. 15, pp. 2546-52, Jul 1997.

[9] R. K. Puri, et al., "Human neurological cancer cells express interleukin-4 (IL-4) receptors which are targets for the toxic effects of IL4-Pseudomonas exotoxin chimeric protein," Int J Cancer, vol. 58, pp. 574-81, Aug 151994.

[10] H. Y. Hong, et al., "Phage display selection of peptides that home to atherosclerotic plaques: IL-4 receptor as a candidate target in atherosclerosis," J Cell Mol Med, vol. 12, pp. 2003-14, Oct 2008.

[11] X. L. Wu, et al., "Tumor-targeting peptide conjugated $\mathrm{pH}$-responsive micelles as a potential drug carrier for cancer therapy," Bioconjug Chem, vol. 21, pp. 208-13, Feb 172010.

[12] J. H. Kim, et al., "Facilitated intracellular delivery of peptide-guided nanoparticles in tumor tissues," J Control Release, Sep 162011. 